\title{
PERMUTATIONS AND TWO SEQUENCES WITH THE SAME CLUSTER SET ${ }^{1}$
}

JAMES A. YORKE

Suppose that $a\left(n \rightarrow a_{n}\right)$ and $b\left(n \rightarrow b_{n}\right)$ are sequences in a compact metric space with distance $d$. Suppose further that $a$ and $b$ have the same set of cluster points $C$. Von Neumann [1, p. 11-12] proved there exists a permutation $\pi$ of the set of positive integers $Z$ such that $d\left(a_{n}, b_{\pi n}\right) \rightarrow 0$. Halmos [2] recently gave an improved proof (and the above statement for compact metric spaces). A discussion of this result may be found in [2]. My purpose is to give a shorter proof than that of Halmos, which used the Schröder-Bernstein Theorem.

Proof. Let $\pi 1=1$. We now construct $\pi n$ inductively for $n>1$, given $\pi 1, \cdots, \pi(n-1)$. Write $Z_{1}$ for $Z$ and $Z_{n}$ for $Z-\{\pi 1, \cdots, \pi(n-1)\}$. Let $\rho(n)=\min Z_{n}$. Let $\pi n$ be the smallest integer in $Z_{n}$ such that

$$
d\left(b_{\pi n}, a_{n}\right) \leqq d\left(a_{n}, C\right)+1 / \rho(n)+d\left(b_{\rho(n)}, C\right) .
$$

Such an element exists: Let $c_{n}$ be chosen in the compact set $C$ so that $d\left(a_{n}, c_{n}\right)=d\left(a_{n}, C\right)$. Then for some $m \in Z_{n}, d\left(b_{m}, c_{n}\right) \leqq 1 / \rho(n)$, since $c_{n}$ is a cluster point of $b$. Then $\pi n=m$ would satisfy (1). To see that $\pi$ is "onto," i.e., $\{\pi 1, \pi 2, \cdots\}=Z$, let us suppose not. Let $q$ $=\min Z-\{\pi 1, \pi 2, \cdots\}$. Then for all $n, \rho(n) \leqq q$, and $N$

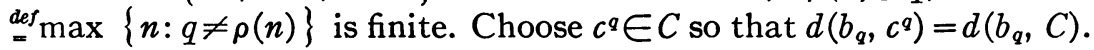
Since $c^{q} \in C, c^{q}$ is a cluster point of $a$, so we may choose $n>N$ such that $1 / q>d\left(a_{n}, c^{q}\right)$. Obviously, $d\left(a_{n}, C\right)+1 / q>d\left(a_{n}, c^{q}\right)$. Since $n>N$, $\rho(n)=q$, and since $\pi n>q, d\left(b_{q}, a_{n}\right)>d\left(a_{n}, C\right)+1 / q+d\left(b_{q}, c^{q}\right)>d\left(a_{n}, c^{q}\right)$ $+d\left(b_{q}, c^{q}\right)$, contradicting the triangle inequality. Hence $\pi$ is onto. Since $\pi n \rightarrow \infty$ and $d\left(a_{n}, C\right) \rightarrow 0$ and $d\left(b_{\pi n}, C\right) \rightarrow 0$, (1) implies $d\left(a_{n}, b_{\pi n}\right)$ $\rightarrow 0$.

\section{REFERENCES}

1. J. von Neumann, Charakterisierung des Spektrums eines Integraloperators, Hermann, Paris, 1935.

2. P. R. Halmos, Permutations of sequences and the Schröder-Bernstein theorem, Proc. Amer. Math. Soc. 19 (1968), 509-510.

Institute for Fluid Dynamics and Applied Mathematics, UNIVERSITY OF MARYLAND

Received by the editors August 14, 1968.

1 Partially supported by National Science Foundation Grant GP-7846. 